\title{
ADAPTACIÓN MULTICULTURAL DE RECURSOS EDUCATIVOS EN ABIERTO: FACTORES DE ÉXITO EN EL PORTAL OPENSCOUT
}

Covadonga Rodrigo-San-Juan, Rodrigo Martín-García y Raquel Arguedas-Sanz

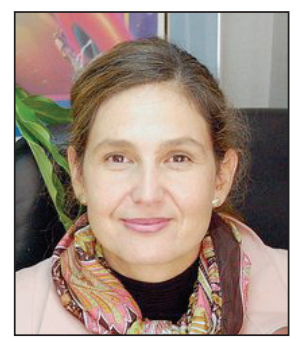

Covadonga Rodrigo-San-Juan es doctora ingeniera de telecomunicación por la Univ. Politécnica de Madrid. Se incorporó al Departamento de Lenguajes y Sistemas Informáticos de la Universidad Nacional de Educación a Distancia (UNED) en 2000 y desde septiembre de 2010 es vicerrectora de Tecnología. Vocal de Aenor en normas de calidad en eLearning (UNE 66181:2004 y 2008), participa en la Task Force Eadtu sobre "Quality in eLearning". Es miembro de la Comisión Ejecutiva en la CRUE TIC, coordinando el grupo "Campus virtuales: servicios integrados y criterios de calidad" y directora de la Cátedra Tecnología y Accesibilidad UNED-Fundación Vodafone España. http://orcid.org/0000-0001-8135-3163

UNED - ETSI Informática, Depto. de Lenguajes y Sistemas Informáticos c/Juan del Rosal, 16. 28040 Madrid, España covadonga@lsi.uned.es

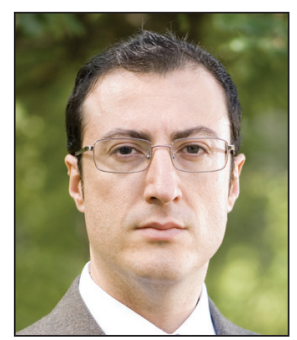

Rodrigo Martín-García es profesor de economía financiera en la Universidad Nacional de Educación a Distancia (UNED) y profesor-tutor en el Instituto de Estudios Fiscales. Licenciado en administración y dirección de empresas por el Colegio Universitario de Estudios Financieros (Cunef) y master en dirección y gestión integral de empresas inmobiliarias y de la construcción (UNED). Entre 2008 y 2012 es vicerrector adjunto de Coordinación de Centros Asociados de la UNED. Áreas centrales de investigación y publicación son: finanzas, estrategia empresarial, innovación educativa y tecnologías aplicadas a la educación superior.

http://orcid.org/0000-0002-9065-7481

UNED - Fac. de Ciencias Económicas y Empresariales, Depto. de Economía de la Empresa y Contabilidad Paseo Senda del Rey 11. 28040 Madrid, España rmarting@cee.uned.es

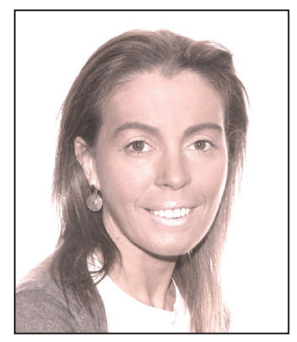

Raquel Arguedas-Sanz es doctora en ciencias económicas y empresariales por la Universidad Nacional de Educación a Distancia (UNED). Premio extraordinario de doctorado y premio Víctor Mendoza 2010 del Instituto de Estudios Económicos. Profesora titular de economía financiera en el Departamento de Economía de la Empresa y Contabilidad de la UNED. Obtuvo la certificación International financial risk manager ${ }^{\mathrm{TM}}$ (FRM) de la Global Association of Risk Professionals. Ha desarrollado su actividad docente e investigadora en finanzas empresariales y gestión y supervisión de riesgos financieros, así como la aplicación de las TIC a la educación.

http://orcid.org/0000-0001-7368-6347

UNED - Fac. de Ciencias Económicas y Empresariales, Depto. de Economía de la Empresa y Contabilidad Paseo Senda del Rey 11. 28040 Madrid, España rarguedas@cee.uned.es

\section{Resumen}

Se expone la experiencia llevada a cabo en la UNED (Universidad Nacional de Educación a Distancia) para extender la utilización de recursos educativos en abierto en el aprendizaje y el desarrollo de competencias profesionales. Con este fin se integró en el proyecto de investigación europeo OpenScout, participando en el diseño, creación, análisis y evaluación de un modelo de aprendizaje informal a partir de un repositorio federado de recursos educativos, sobre el que se han utilizado y reutilizado los materiales existentes y se han generado nuevos objetos en un entorno creativo, colaborativo, de ámbito internacional y multilingüe. El proceso de adaptación de recursos se ha analizado en el contexto cultural español. Se muestran las fortalezas de la plataforma OpenScout vinculadas a la re-utilización de contenidos en abierto dentro del proceso educativo y la capacidad de integración en comunidades virtuales más amplias, buscando las sinergias derivadas del uso combinado con las redes sociales más conocidas.

\section{Palabras clave}

Aprendizaje multicultural, Recursos educativos en abierto, Tecnologías de aprendizaje, Redes sociales, Aprendizaje colaborativo, Investigación cualitativa. 


\title{
Title: Multicultural adaptation of open educational resources: factors in the OpenScout portal's success
}

\begin{abstract}
This paper outlines the experience developed in the UNED (Spanish National University of Distance Education) to extend the use of Open Educational Resources for business and management education. UNED joined the European research project OpenScout --which offers value-added tools and services for learners, educators and technology providers- and participated in the design, creation, analysis and evaluation of a model of informal learning. OpenScout draws from a federated repository of educational resources, using and re-using the existing materials and generating new ones, in a creative, collaborative, multilingual international initiative. The cultural adaptation process has been analyzed in the Spanish context. The main strengths of OpenScout, linked to the re-use of open contents in the educational process, are described. In addition, we discuss the project's capacity for integration into larger virtual communities, seeking synergies from combined use with the most popular social networks.
\end{abstract}

\section{Keywords}

Multicultural education, Open educational resources (OER), Learning technologies, Social networks, Cooperative learning, Qualitative research.

Rodrigo-San-Juan, Covadonga; Martín-García, Rodrigo; Arguedas-Sanz, Raquel (2013). “Adaptación multicultural de recursos educativos en abierto: factores de éxito en el portal OpenScout". El profesional de la información, noviembrediciembre, v. 22, n. 6, pp. 537-544.

http://dx.doi.org/10.3145/epi.2013.nov.06

\section{Introducción}

El trabajo que aquí se presenta es parte de una iniciativa europea para la popularización del uso de los recursos en abierto, en un entorno colaborativo que favorece la generalización del trabajo en comunidad y la difusión y compartición de elementos a través de redes sociales. Este objetivo se ha materializado en el proyecto OpenScout ${ }^{1}$ que ha involucrado a 18 universidades e institutos de investigación de 14 países, creando un portal web que provee utilidades específicas para búsquedas, etiquetado semántico, etiquetado sobre competencias y re-publicación con posibilidades de adaptación multicultural y difusión social de contenidos en abierto en temas relacionados con administración y gestión empresarial (Kalz et al., 2010). Su carácter original de plataforma federada de repositorios de recursos educativos en abierto (OER, open educational resources), pretende desarrollar la función que Parodi et al. (2010) denominan "conector" para el acceso de los usuarios a los contenidos, que combina servicios fuente heterogéneos para producir nuevos servicios orientados a la mejora de entornos formales y no formales.

En la propia UNED existían algunos trabajos previos en modelos blended (Read et al., 2011), así como la participación de la institución en el portal OCW (OpenCourseWare) ${ }^{2}$, si bien siempre haciendo uso de materiales contenidos en sus propios repositorios. La intervención en el proyecto OpenScout, sin embargo, supone una contribución a una estructura federada, integrada en una comunidad multicultural y completamente online.

El presente trabajo se estructura como sigue: el apartado 2 analiza las iniciativas y estrategias en materia de recursos educativos en abierto en el entorno educativo, como base para la utilización, intercambio y reutilización de contenidos, así como su adaptación multicultural. En el apartado 3 se describen las grandes líneas del proyecto OpenScout y la contextualización del trabajo de la UNED, mientras que el apartado 4 detalla el estudio cualitativo formado por los pilotos a gran escala del proyecto con estudiantes de tres asignaturas en diferentes cursos y planes de estudio, sobre los cuales se ha realizado una encuesta. El apartado 5 expone el análisis de los resultados de este trabajo. Finalmente, en el apartado 6 se muestran las principales conclusiones obtenidas en todo el proceso.

Extender la utilización de recursos educativos en abierto (OER) en el estudio y el desarrollo de competencias profesionales

\section{Los OERs en el contexto educativo actual}

El movimiento OER se inició en 2001 cuando el Massachusetts Institute of Technology (MIT) se planteó colocar en abierto todos los materiales de sus cursos de grado y postgrado en un proyecto conjunto con las Fundaciones William and Flora Hewlett y Andrew W. Mellon (MIT OCW - OpenCourseWare). Sin embargo, el término OER como tal fue acuñado un año más tarde por la Unesco en el Primer foro mundial sobre recursos educativos de libre acceso, como recursos de libre acceso que están a disposición de una comunidad abierta para ser compartidos, re-elaborados y reutilizados una y otra vez. La Declaración de París (Unesco, 2012) sobre los OERs en el décimo aniversario de la acuñación del término marcó un hito, llamando la atención a todos los gobiernos del mundo para que dotasen de materiales educativos de uso público con licencias abiertas y financiados por el Estado.

En Santos-Hermosa, Ferran-Ferrer y Abadal (2012) se puede encontrar una excelente revisión de la historia, caracte- 
rísticas, desarrollos, proyectos y líneas de investigación en esta materia y cómo el repositorio inicial del OCW con el que se comenzó de una forma más o menos expositiva se ha convertido hoy en día en un proyecto amplísimo a nivel mundial en constante crecimiento, incluyendo a instituciones de 49 países $^{2}$ entre ellos España. Esta nueva infraestructura de participación abierta está ayudando realmente a moldear una nueva cultura del aprendizaje online (Daniel; Uvalić-Trumbić, 2012), cada vez más interactivo, social y accesible, como se refrenda últimamente en el caso de los moocs (massive open online courses) (Daniel, 2012).

Son continuos los trabajos de investigación en búsqueda del modelo de negocio en el uso de los OERs (Butcher; Hoosen, 2012; De-Langen, 2012) y en el planteamiento de los mismos como un instrumento para enriquecer el capital de las organizaciones de manera eficaz en términos de coste (Andreatos, 2011). Pero además se ha fomentado una cultura de intercambio que proporciona un nuevo y potente instrumento docente contribuyendo a cambiar la cultura del profesor del "este curso es mío" a "este contenido es para compartir y reutilizar". Davis et al. (2010) buscan impulsar la compartición de recursos como base para crear una masa crítica suficiente capaz de facilitar el proceso docente. Son experiencias que demuestran que ya se ha fomentado una cultura de aprendizaje en la que tanto el capital intelectual (contenido) como el capital humano (talento) se entrelazan formando una espiral de enriquecimiento.

El proyecto OpenScout pretende ahondar en este contexto, resaltando las especiales características de los OERs y los repositorios federados entre distintas instituciones, para constituir un punto de partida prometedor en las deseables colaboraciones internacionales dentro el ámbito educativo (Pirkkalainen et al., 2010). Por su parte, BuenoDe-la-Fuente y Hernández-Pérez (2011) proponen un conjunto de estrategias de éxito en la creación de repositorios de contenidos digitales educativos en las universidades, haciendo hincapié en las barreras socio-culturales asociadas al intercambio y la reutilización de materiales educativos.

No obstante, para impulsar la participación de manera colaborativa en la generación y adaptación de contenidos existentes se pueden utilizar también las redes sociales de comunicación, como así se ha tratado en OpenScout. Terrase et al. (2012), integrantes del proyecto, concluyen que el análisis detallado de los procesos permite explotar el potencial de las aportaciones individuales y de las colaboraciones y redes de trabajo, y la generalización del uso y la reutilización de los recursos en abierto, lo que proporciona unas ventajas que se estructuran en dos bloques: 1) institucionales: compartición y difusión del conocimiento, mayor visibilidad y reconocimiento internacional, y mayor transparencia del proceso de
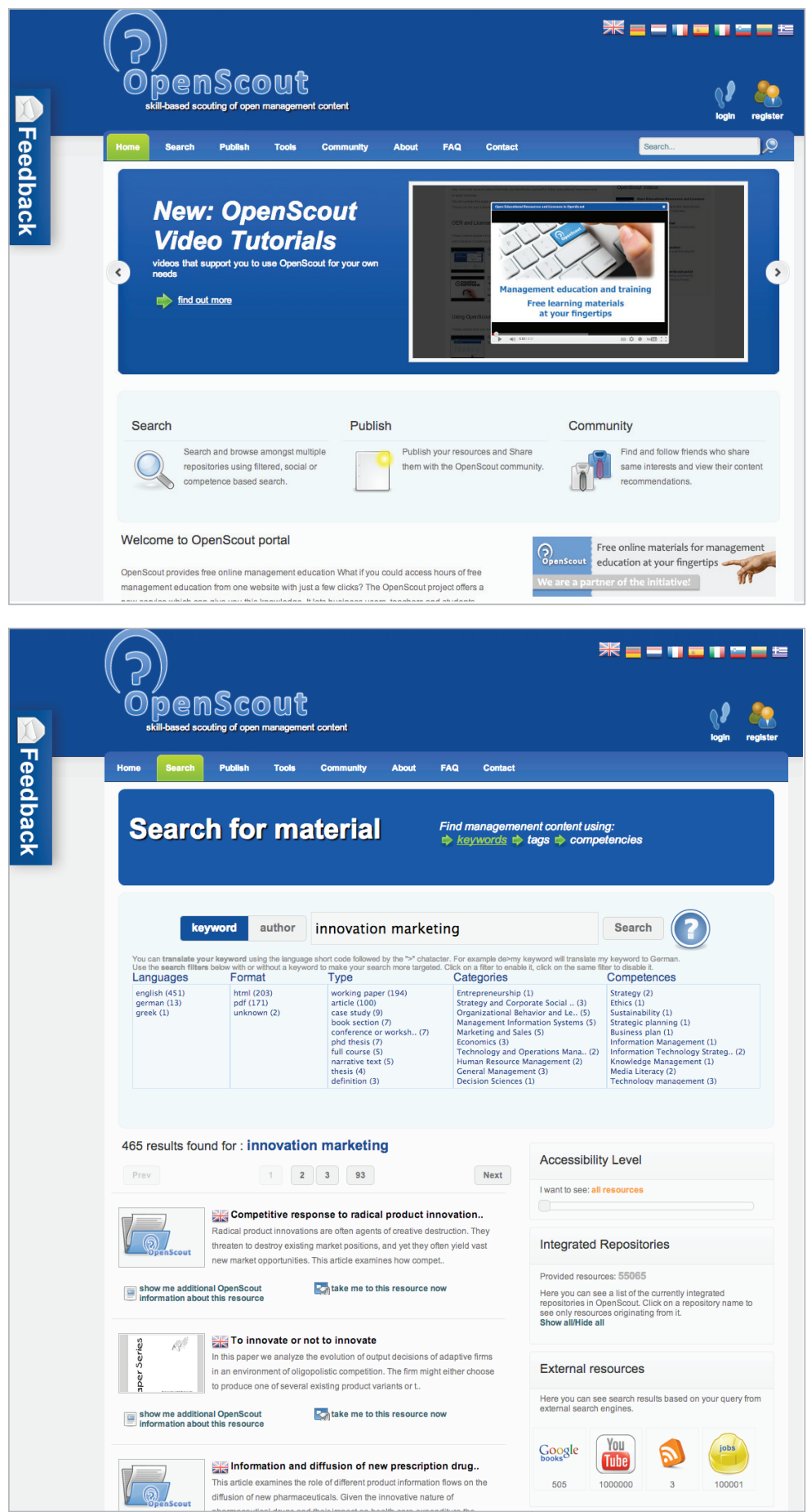

Figura 1. Funciones de OpenScout

http://learn.openscout.net 
Tabla 1. Descripción de los escenarios de OpenScout

\begin{tabular}{|c|c|c|c|}
\hline $\begin{array}{l}\text { Escenario de } \\
\text { uso. } \\
\text { Caracterización }\end{array}$ & $\begin{array}{c}\text { Escenario } 1 \\
\text { Acercamiento a la plataforma }\end{array}$ & $\begin{array}{c}\text { Escenario } 2 \\
\text { Adaptación y reutilización de recursos } \\
\text { educativos }\end{array}$ & $\begin{array}{c}\text { Escenario } 3 \\
\text { Internacionalización }\end{array}$ \\
\hline Objetivos & $\begin{array}{l}\text { - Aprender cómo adaptar y reutilizar los } \\
\text { recursos educativos de acuerdo con } \\
\text { sus necesidades de aprendizaje. } \\
\text { - Contribuir a la biblioteca de herra- } \\
\text { mientas por medio de ejemplos, infor- } \\
\text { mación, recomendaciones, etc. }\end{array}$ & $\begin{array}{l}\text { - Aprender a realizar un trabajo de colaboración } \\
\text { con objetivos y plazos explícitos. } \\
\text { - Aplicar las competencias adquiridas anterior- } \\
\text { mente. } \\
\text { - Propuesta y utilización de herramientas y aplica- } \\
\text { ciones adicionales a las ofertadas por la platafor- } \\
\text { ma, para trabajo autónomo y en grupo. } \\
\text { - Coordinación del trabajo con terceros. } \\
\text { - Colaboración con otros grupos. } \\
\text { - Desarrollo y entrega de trabajos a medida. }\end{array}$ & $\begin{array}{l}\text { Todos los correspondientes al Es- } \\
\text { cenario } 2 \text { y, además: } \\
\text { - Adaptar los materiales a las dife- } \\
\text { rencias de idioma, la cultura y de } \\
\text { otro tipo, derivados de la globa- } \\
\text { lización y la internacionalización }\end{array}$ \\
\hline Actividades & $\begin{array}{l}\text { Aprendizaje: búsqueda, calificación, eti- } \\
\text { quetado, compartición, redes sociales. }\end{array}$ & $\begin{array}{l}\text { Trabajo colaborativo para el desarrollo de una cul- } \\
\text { tura de uso de recursos en abierto y para la gene- } \\
\text { ración de nuevos recursos. }\end{array}$ & $\begin{array}{l}\text { Todas las correspondientes al Es- } \\
\text { cenario } 2 \text {, en un entorno interna- } \\
\text { cional. }\end{array}$ \\
\hline $\begin{array}{l}\text { Competencias } \\
\text { que desarrolla }\end{array}$ & $\begin{array}{l}\text { - Gestión autónoma y autorregulada } \\
\text { del trabajo. } \\
\text { - Desarrollar el uso de herramientas y } \\
\text { aplicaciones informáticas. } \\
\text { - Competencias en la búsqueda, iden- } \\
\text { tificación e interpretación de fuentes } \\
\text { de información relevante. }\end{array}$ & $\begin{array}{l}\text { - Capacidad para trabajar en equipo: liderazgo, } \\
\text { capacidad para coordinar el trabajo con otros. } \\
\text { - Fomentar la búsqueda y el uso de aplicaciones } \\
\text { informáticas para resolver los problemas y ne- } \\
\text { cesidades. } \\
\text { - Comunicación y redacción. } \\
\text { - Habilidades relacionadas con el uso de aplica- } \\
\text { ciones informáticas utilizadas en la gestión em- } \\
\text { presarial. } \\
\text { - Desarrollo de habilidades para realizar estudios } \\
\text { adicionales en el campo de la administración y } \\
\text { la gestión empresarial con un alto grado de au- } \\
\text { tonomía. }\end{array}$ & $\begin{array}{l}\text { Todas las correspondientes al Es- } \\
\text { cenario } 2 \text { y, además: } \\
\text { - Desarrollar la capacidad para } \\
\text { adaptar contenidos al idioma, } \\
\text { cultura y necesidades propias. }\end{array}$ \\
\hline
\end{tabular}

Integra una treintena de repositorios con contenidos con licencias Creative Commons e incorpora documentos en múltiples formatos (textos, presentaciones, hojas de cálculo, audio y video, entre otros) en múltiples idiomas (alemán, español, finlandés, francés, inglés, italiano, lituano...).

Los servicios prestados por OpenScout (Holtkamp et al., 2011) son:

- Búsqueda y recuperación de material procedente de un gran conjunto federado de repositorios educativos abiertos con la integración de estos servicios de búsqueda en sistemas externos.

- Navegación por los contenidos en base a las competencias y a la resolución de problemas conectados con dichas competencias.

- Aglutinación de grupos de interés, y formación de comunidades de aprendizaje informal.

- Colaboración, incluyendo la reutilización y la reorientación de los contenidos para atender a las necesidades existentes e incorporación de información adicional como competencias profesionales asociadas.

- Revisión y recuperación de contenidos para una rápida adaptación a un contexto multicultural y multilingüe.

La participación de la UNED (socio español del proyecto) se enmarcó en los paquetes de trabajo WP4 (formación de comunidades e integración de grupos de usuarios) y WP6 (aplicación de escenarios de adaptación, etiquetación y reutilización a gran escala y su evaluación), con un objetivo doble: transmitir el interés de la utilización de los recursos en abierto e impulsar la participación de manera colaborativa en la generación y adaptación de contenidos existentes. El valor de la UNED como socio del proyecto residió precisamente en el planteamiento de los estudios de caso en entornos considerados masivos (large scale trials) dado que cuenta con asignaturas con centenares y miles de participantes y con varias comunidades activas de profesores e investigadores dentro de la plataforma de la universidad a los que era fácil acceder.

\section{Utilizar y reutilizar materiales educativos y la generación de nuevos objetos den- tro de un entorno creativo, colaborativo, de ámbito internacional y multilingüe}

\section{Realización de los estudios de caso a gran escala}

El proyecto OpenScout ha estado en origen orientado a diversos colectivos: docentes, estudiantes, bibliotecarios y profesionales, tanto procedentes de pequeñas y medianas empresas como de grandes empresas y organizaciones. Los estudios de caso (pilotos) propuestos en la UNED se realizaron con estudiantes de varios cursos y distintos planes de estudio (licenciatura, diplomatura y grado), así como una parte de los docentes encargados de las asignaturas, teniendo todos ellos como punto de coordinación las plataformas docentes de la universidad (WebCT o $\operatorname{dot} L R N$ según el curso y plan). La comunicación entre los docentes implicados y los grupos de estudiantes tuvo lugar principalmente a través de foros, noticias, etc., así como con el uso del correo electrónico personal y la red social Facebook.

Para la consecución de los objetivos del proyecto, se diseña- 
ron tres contextos de uso que, a su vez, determinaron tres escenarios con dinámicas de trabajo diferenciadas (tabla 1).

Para las actividades programadas en los escenarios se contó con estudiantes voluntarios. Todos ellos realizaron el escenario 1 y posteriormente fueron repartidos en grupos para participar en los escenarios 2 y 3 , trabajando colaborativamente sobre los recursos. Asimismo todos los participantes tuvieron que cumplimentar una encuesta final, para obtener datos sobre sus perfiles de usuario e impulsar la retroalimentación sobre el trabajo realizado, avanzando en el proceso de mejora continua propuesto en el proyecto OpenScout sobre su portal de servicios. En este sentido se pidieron valoraciones de usabilidad y navegación tanto del portal web como de los sucesivos apartados: herramientas de adaptación de contenidos, plataforma de comunidades virtuales, servicios de conexión con redes sociales...

La encuesta tenía 43 preguntas sobre el perfil de los participantes, expectativas de uso del portal y percepción inicial del mismo, utilización y reutilización de materiales existentes, aplicación de las herramientas propuestas o de otras nuevas, y valoración global de las utilidades y herramientas OpenScout. Un total de 70 estudiantes ${ }^{2}$ completó todas las actividades, incluida la encuesta, conforme a la distribución que se presenta en la tabla 2 de las asignaturas implicadas. La disparidad de participación entre las asignaturas respondió al volumen de matrícula de cada una, el curso y el plan de estudio en que se integraban.

En la observación de la muestra se comprueba que hay mayor proporción de mujeres (60\%) que de hombres (40\%). Por otra parte, la edad media de los participantes es consistente con la estructura del alumnado de la UNED, con una mayor frecuencia para el intervalo 25-39 años.

Por otra parte, más de la mitad contaba con estudios universitarios previos. El $29 \%$ del total eran diplomados o licenciados; el 19\% había realizado estudios de posgrado (el $4 \%$ había concluido un MBA); incluso, había un participante con un doctorado finalizado. Existe constancia de que una proporción relevante de ellos compatibilizaba obligaciones profesionales, familiares, etc., con sus estudios ${ }^{1}$, lo que proporcionó al proyecto OpenScout una doble óptica académica y profesional.

\section{Resultados de las pruebas masivas de uso del portal OpenScout}

Para conocer la motivación, intención de uso y opinión de los usuarios en la encuesta final se propusieron dos tipos de pregunta:

a) categorizadas, a fin de poder trasladar a lenguaje numérico opiniones e ideas básicas para la investigación, por medio de la jerarquización de ideas, nivel de acuerdo o desacuerdo, intencionalidad, etc.;

b) abiertas, para que los estudiantes pudieran expresar libremente sus opiniones, proporcionando un importante volumen de información acerca de los puntos fuertes y aspectos a mejorar de la plataforma.

A continuación se exponen los principales resultados:

\section{Expectativas de uso del portal y percepción inicial del mismo}

Los usuarios plantearon una utilización muy práctica, orientada en todo momento a objetivos tangibles y próximos: obtención de conocimientos (selección pormenorizada de recursos afines) y uso de herramientas de adaptación a competencias ( $86 \%$ de los encuestados), conocimiento en materia empresarial (80\%) y búsqueda de información para la preparación de exámenes (79\%). Otros objetivos, como son la re-edición de materiales para uso por parte de terceros y el trabajo colaborativo mostraron tener una importancia muy inferior para estos usuarios.

\section{Utilización de la plataforma OpenScout}

El portal OpenScout ofrece una infraestructura integrada que genera múltiples sugerencias y campos de trabajo (figura 2). Se provee acceso federado a los repositorios de recursos, a la búsqueda y enriquecimiento de contenidos y a la manipulación directa de los metadatos asociados. Por todo ello, la aportación de los participantes fue especialmente útil para detectar errores, para la prospección de nuevos enfoques, aplicaciones y para la selección de las herramientas que pueden resultar de mayor interés en el trabajo colaborativo con este tipo de OER.

Los OERs enriquecen el capital de las organizaciones en términos de coste, y los metadatos son la pieza clave para conseguir la mejor organización, búsqueda y recuperación de la información

Las actividades de utilización directa de OER para el aprendizaje orientaron los comentarios y sugerencias hacia temas de accesibilidad, facilidad de navegación y primeras actuaciones en el portal (uso del motor de búsqueda y valoración de los resultados obtenidos). Eso sí, la principal vía de mejora sugerida fue el incremento en el número de recursos, ya que el volumen de elementos federados se consideró insuficiente.

También se preguntó sobre adaptación y reutilización de recursos educativos, tanto en un contexto nacional como multicultural y de contextualización en un marco internacional. La mayoría de usuarios consideró que es fácil publicar nuevos documentos, que se comprenden bien los metadatos de publicación y que no resultan excesivos en cantidad, por lo que les pareció una la valoración de la plataforma de publicación fue positiva. Sin embargo, existían dudas acerca de cómo asignar un recurso a un dominio de clasificación, de la asignación de competencias adecuadas, así como de 




Figura 2. Utilidad percibida de los distintos usos del portal OpenScout la plataforma educativa virtual de referencia, las comunidades generales y comunidades propias en el portal OpenScout $y$, de forma mayoritaria, el correo electrónico. En cualquier caso, es posible anticipar un prometedor futuro para este trabajo colaborativo en el ámbito profesional, ya que son muchos los usuarios que comprenden y aprueban el funcionamiento del trabajo en grupo dentro de las comunidades y muestran intención de uso en el futuro. Una vez solventado aquello que se percibe como problemas de seguridad de las redes sociales, una mala adjudicación del tipo de licencia de copyright. Por todo ello, ocurre que en algunos casos la tarea de la etiquetación semántica y de competencias, imprescindible para la clasificación de los recursos, resultó algo tediosa y compleja para los usuarios en el momento de la aportación de documentos al repositorio.

La aportación de los participantes ha sido útil en la detección de errores, prospección de nuevos enfoques, y aplicaciones, y selección de las herramientas

Por otra parte, con el fin de comprobar hasta qué punto el aspecto social puede condicionar la formación y habilidades de los propios usuarios del portal OpenScout en materia de economía, administración y dirección de empresas, se incorporó a la encuesta -a propuesta de integrantes del proyecto (Kalz et al., 2010)- un bloque de preguntas sobre la interacción en la generación y publicación de nuevos OERs y el fomento de la compartición y reutilización. Se probó de este modo que las habilidades y conocimientos profesionales son, en parte, adquiridos a través de relaciones sociales dentro y fuera de sus organizaciones, proveedores, clientes, gerentes de bancos, además de las provenientes de la educación universitaria, la afiliación profesional, los padres y los mentores.

En este bloque de preguntas, los usuarios manifestaron sus reservas al no haber intervenido en la configuración de los grupos, por un factor en principio inesperado: fue considerado un obstáculo para conservar la confidencialidad de sus datos personales, por lo que el uso de la plataforma que se les facilitó fue limitado, en favor de otras formas de comunicación. Los instrumentos de socialización más habituales fueron los grupos cerrados de usuarios creados dentro de yoría recomendaría la comunidad OpenScout a compañeros y amigos y manifiesta interés en colaborar con terceros en esta plataforma para desarrollar nuevos recursos.

Las herramientas de trabajo constituyen una parte relevante del valor añadido de la plataforma. Desde OpenScout se seleccionó un conjunto de aplicaciones gratuitas, clasificadas en dos grupos: de edición de materiales escritos y gráficos, que facilitan la incorporación y/o re-edición de recursos; y, en segundo lugar, de comunicación, para fomentar el flujo de información, crear relaciones de trabajo, estimular la colaboración y la creación de comunidades con intereses comunes. El 92\% de los participantes utilizó alguna de todas las propuestas; el $85 \%$, al menos 2 y el $63 \% 3$ ó más aplicaciones.

\section{Valoración global y resultados obtenidos por la utili- zación de OpenScout}

Pese a haber obtenido un importante nivel de satisfacción por parte de los usuarios, se buscaron líneas de mejora en todos los aspectos de usabilidad de la plataforma. Las contribuciones se obtuvieron de las preguntas abiertas del cuestionario y de la dinámica de trabajo a lo largo del periodo de prueba.

En primer lugar, se puso de manifiesto la problemática del idioma. Este punto se explicitó por parte de un número relevante de usuarios: fue muy recurrente la percepción de la utilización de lenguas distintas a la materna como una barrera a superar. Se recibieron aportaciones de gran utilidad para perfeccionar y completar la traducción del portal y el requerimiento más habitual fue la incorporación de documentos en español sobre temáticas concretas.

Un segundo grupo de sugerencias fue el referido a cuestiones técnicas, debido fundamentalmente a la variedad de navegadores y programas para visualizar los recursos. Además, se puso de manifiesto la creciente exigencia de los usuarios 
con el buen funcionamiento y la velocidad del portal.

En lo relativo a la apariencia del portal (look \& feel), se obtuvieron múltiples recomendaciones de los participantes. La apariencia novedosa, la maniobrabilidad entre páginas y la flexibilidad de edición de los perfiles de usuario fueron algunos de los aspectos más valorados.

Por el momento, dado el escaso desarrollo de las plataformas de recursos en abierto frente a otras fuentes de contenidos, la principal alternativa para la obtención de documentación son los buscadores genéricos (Google, Bing, Ask Search, etc.). Si bien, se empieza a detectar un cambio de tendencia en los procesos de búsqueda entre aquellos que ya han utilizado OpenScout.

Sin embargo, el objetivo de OpenScout y, por extensión de las plataformas de OER, no debe ser desplazar a los buscadores tradicionales como alternativa de obtención de recursos genéricos, sino ofrecer una alternativa eficaz y complementaria para incentivar la adaptación multicultural de contenidos y mejorar las búsquedas a través del enriquecimiento de la semántica asociada a los recursos (etiquetado mediante metadatos), tanto en el ámbito educativo como en el profesional.

Diseñar y analizar un modelo de aprendizaje informal a partir de un repositorio federado de recursos educativos

\section{Conclusiones}

El proyecto OpenScout proporciona una plataforma al servicio de todo tipo de usuarios, para compartir recursos en abierto, con una filosofía de diseño centrada en el usuario que se refrenda en todas las pruebas realizadas. Así, la aportación de los usuarios es múltiple y distintiva frente a otras opciones -como podría ser Google scholar- puesto que en OpenScout los usuarios participan en la creación de nuevos recursos en abierto, colaboran en la adaptación idiomática, cultural o simplemente al contexto de uso, actúan como prescriptores del servicio y lo que es más, ayudan a identificar las pautas para el buen funcionamiento de la plataforma y el ajuste del servicio a sus necesidades.

Además, la retroalimentación de los usuarios ha permitido identificar que es fundamental contar con una amplia base de recursos (la federación de repositorios es por tanto clave) y que los aspectos de usabilidad y accesibilidad web (apariencia del portal, uso intuitivo y funcionalidad) y la flexibilidad ocupan el segundo puesto por relevancia en las recomendaciones. En concreto, se valora muy positivamente la prescripción y el incentivo de uso de herramientas para el aprendizaje.

Por el momento, el uso de las redes sociales vinculado al proceso educativo se encuentra en un estadio inicial. En general, los usuarios del portal OpenScout han mostrado preferencia a integrarse en grupos pequeños frente a las comunidades amplias. Esto se debe, según manifiestan, a sus reservas por lo que perciben como problemas de confidencialidad y lleva a preferir medios alternativos de sociali- zación o comunidades cerradas. Será interesante conocer la evolución de este aspecto en el futuro, ya que hay una importante intención de uso, existen redes sociales profesionales de contactos (como Linkedln o Xing) que los usuarios conocen y en las que participan. Además, consideran que la integración en grupos y comunidades globales contribuirá a conseguir las metas de aprendizaje en su futuro académico y profesional.

Asimismo, el trabajo presentado demuestra que OpenScout ha contribuido a proporcionar nuevas ideas para el enriquecimiento de los procesos de aprendizaje informal. En concreto, se ha puesto de manifiesto la utilidad de la introducción paulatina de contenidos en otras lenguas al proceso docente, para acercar al alumno a la búsqueda y adaptación multicultural de recursos internacionales y mejorar así su futuro profesional y la adaptación a un entorno global de aprendizaje, en el que serán clave la interconectividad entre usuarios y la interoperabilidad de los soportes.

\section{Notas}

\section{1. http://ocw.innova.uned.es}

\section{2. http://www.ocwconsortium.org}

3. No es posible mostrar porcentajes en lo relativo a este punto, ya que no se planteó esta pregunta de manera explícita en la encuesta, pero se deduce de las respuestas ofrecidas a las preguntas abiertas.

\section{Agradecimientos}

El proyecto OpenScout ha sido financiado por la Comisión Europea a través del programa eContentplus ECP 2008 EDU 428016 (cf. http://www.openscout.net).

\section{Bibliografía}

Andreatos, Antonios (2011). "The use of open educational resources in intra-organisational elearning and continuing education". En: Procs of the $10^{\text {th }}$ European conf on e-learning, v. 1-2. Brighton Business School, Brighton, Inglaterra, 10-11 Nov., pp. 23-33.

Bueno-De-la-Fuente, Gema; Hernández-Pérez, Tony (2011). "Estrategias para el éxito de los repositorios institucionales de contenido educativo en las bibliotecas digitales universitarias". BiD: Textos universitaris de biblioteconomia i documentació, n. 26, julio.

http://bid.ub.edu/26/bueno2.htm

Butcher, Neil; Hoosen, Sarah (2012). Exploring the business case for open educational resources. Commonwealth of Learning, Sept. ISBN: 9781894975599

http://www.col.org/resources/publications/Pages/detail. aspx?PID=421

Daniel, John (2012). "Making sense of moocs: musings in a maze of myth, paradox and possibility". Journal of interactive media in education, North America, $3 \mathrm{Dec}$.

http://jime.open.ac.uk/jime/article/view/2012-18

Daniel, John; Uvalić-Trumbić, Stamenka (2012). “Open educational resources (OER): the coming of age of ICT in education?". Speech to eLearning Korea, 12 Sept. http://sirjohn.ca/wordpress/?page_id $=29$ 
Davis, Hugh; Carr, Leslie; Hey, Jessie; Howard, Yvonne; Millard, David; Morris, Debra; White, Su (2010). "Bootstrapping a culture of sharing to facilitate open educational resources". IEEE Transactions on learning technologies, v. 3, n. 2, pp. 96-109.

http://dx.doi.org/10.1109/TLT.2009.34

De-Langen, Frank (2012). "Business models for OER. A community based approach". First Unisa intl open distance learning conf, Pretoria, South Africa, 6 Sept.

Holtkamp, Philipp; Pawlowski, Jan; Pirkkalainen, Henri; Schwertel, Uta (2011). OpenScout annual intermediate public report (01.09.2010 - 31.08.2011).

http://www.openscout.net

Kalz, Marco; Specht, Marcus; Nadolski, Rob; Bastiaens, Yves; Leirs, Nele; Pawlowski, Jan (2010). “OpenScout: competence based management education with community-improved open educational resources". In: Halley, S. (editor). Procs of the $17^{\text {th }}$ Edineb conf. Crossing borders in education and work-based learning, 9-11 June, pp. 137-146. Londres: FEBA ERD Press.

http://dspace.ou.nl/handle/1820/2452

Parodi, Elisabetta; Dicerto, Michele; Schwertel, Uta (2010). "A connector for open educational resources from repositories to LCMSs and social networks". En: Procs of special track "Tools and resources for lifelong learning", Intl conf on interactive computer aided learning (ICL 2010). Hasselt, Bélgica, pp. 1115-1122.

http://www.icl-conference.org/dl/proceedings/2010/ contributions/Contribution339.pdf

Pirkkalainen, Henri; Thalmann, Stefan; Pawlowski, Jan; Bick, Markus; Holtkamp, Philipp; Ha, Kyung-Hun (2010).
"Internationalization processes for open educational resources". En: Tyrväinen, P.; Jansen, S; Cusumano, M. A. (editors). Workshop on competencies for the globalization of information systems in knowledge-intensive settings, $1^{\text {st }}$ Intl conf on software business, Icsob 2010, Jyväskylä, Finland, 21-23 June, pp. 57-64.

http://iwi.uibk.ac.at/download/downloads/Publikationen/ ICSOB.pdf

Read, Timothy; Ros, Salvador; Pastor, Rafael; Hernandez, Roberto; Rodrigo, Covadonga (2011). "The generation and exploitation of open educational resources in virtual attendance in UNED". Advances in new technologies, interactive interfaces, and communicability: $1^{\text {st }}$ int l conf, v. 6616, pp. 132-141. http://dx.doi.org/10.1007/978-3-642-20810-2_14

Santos-Hermosa, Gema; Ferran-Ferrer, Núria; Abadal, Ernest (2012). "Recursos educativos abiertos: repositorios y uso". El profesional de la información, v. 21, n. 2, pp. 136-145. http://dx.doi.org/10.3145/epi.2012.mar.03

Terrasse, Christophe; Marinova, Boriana; Greller, Wolfgang; Bitter-Rijpkema, Marlies; Schwertel, Uta (2012). "Opening up! How to take full advantage of open educational resources (OER) for management education". En: Rienties, Bart; Daly, Peter; Reeb-Gruber, Sandra, et al. Procs of the $19^{\text {th }}$ Edineb conf the role of business education in a chaotic world. Haarlem, Netherlands: FEBA ERD Press. ISBN: 9789081372756

Unesco (2012). Declaración de París de 2012 sobre los REA. Congreso mundial sobre los recursos educativos abiertos (REA). Unesco, París, 20-22 de junio.

http://www.unesco.org/new/fileadmin/MULTIMEDIA/HQ/ $\mathrm{Cl} / \mathrm{Cl} /$ pdf/Events/Paris\%200ER\%20Declaration_01.pdf

\section{Próximos temas centrales}

Enero 2014

Marzo 2014

Mayo 2014

Julio 2014

Septiembre 2014

Noviembre 2014

Enero 2015
Documentación audiovisual

Políticas de información

Visualización de información

Humanidades digitales

Big data y analítica web

Marketing

Web semántica

Los interesados por favor consulten detenidamente las Normas para autores:

http://www.elprofesionaldelainformacion.com/autores.html

y luego envíen sus artículos a través del gestor de manuscritos OJS de la plataforma del Recyt: http://recyt.fecyt.es/index.php/EPI/index 